\title{
Genetic variations in open reading frame 2 gene of porcine circovirus type 2 isolated in Korea during 2016-2017
}

\author{
Kiju Kim ${ }^{1}$, Jong-Young Choi ${ }^{1,2}$, Kwang-Soo Lyoo ${ }^{3}$, Tae-Wook Hahn ${ }^{1, *}$ \\ ${ }^{1}$ College of Veterinary Medicine \& Institute of Veterinary Science, Kangwon National University, Chuncheon 24341, Korea \\ ${ }^{2}$ Dodam Veterinary Clinic, Seoul 04716, Korea \\ ${ }^{3}$ Korea Zoonosis Research Institute, Chonbuk National University, Iksan 54531, Korea
}

(Received: June 21, 2018; Revised: July 13, 2018; Accepted: July 18, 2018)

\begin{abstract}
The capsid protein of porcine circovirus type 2 (PCV2) encoded by open reading frame 2 (ORF2) is important for neutralizing activity against PCV2 infection. This study investigated the heterogeneity of the ORF2 gene of PCV2 isolated in Korea during 2016-2017. The results revealed that PCV2d is currently the predominant genotype. Moreover, comparison of ORF2 from 17 PCV2 isolates revealed $88.3-100 \%$ homology at the nucleotide (deduced amino acid $86.3-100 \%)$ level. Interestingly, $61.5 \%(8 / 13)$ of the PCV2d isolates had glycine at position 210 . These data provide a useful information for PCV2 epidemiology in Korea.
\end{abstract}

Keywords: capsid proteins, genetic variation, phylogenetic analysis, porcine circovirus type 2

Porcine circovirus type 2 (PCV2) is a small, nonenveloped, circular single-stranded DNA virus belonging to the genus Circovirus of the family Circoviridae [16]. PCV1 is nonpathogenic but pathogenic PCV2 is the primary etiological agent of PCV2-associated diseases (PCVAD) that include post-weaning multisystemic wasting syndrome (PMWS) and porcine dermatitis and nephropathy syndrome, which both cause major economic losses in the swine industry worldwide. PCV3 was recently reported as a novel porcine circovirus and its pathogenesis is still unknown [6].

Table 1. Porcine circovirus type 2 (PCV2) isolates analyzed in this study

\begin{tabular}{llccccc}
\hline \hline PCV2 strain & Geographic origin & $\begin{array}{c}\text { Year of the } \\
\text { isolation }\end{array}$ & $\begin{array}{c}\text { Genome size } \\
(\mathrm{nt})\end{array}$ & $\begin{array}{c}\text { ORF2 size } \\
(\mathrm{nt})\end{array}$ & $\begin{array}{c}\text { Genotype } \\
\text { GenBank accession } \\
\text { number }\end{array}$ & $\begin{array}{c}\text { Gen } \\
\text { HID5697 }\end{array}$ \\
HID5701 & Nonsan & 2016 & 1768 & 702 & PCV2b & KY810319 \\
HID5703 & Sangju & 2016 & 1767 & 705 & PCV2d & KY810320 \\
HID5705 & Yeoju & 2016 & 1768 & 702 & PCV2a & KY810321 \\
HID5707 & Sejong & 2016 & 1768 & 702 & PCV2a & KY810322 \\
HID5709 & Sejong & 2016 & 1767 & 705 & PCV2d & KY810323 \\
HID5715 & Jeju & 2016 & 1767 & 705 & PCV2d & KY810324 \\
HID5733 & Cheongyang & 2016 & 1767 & 705 & PCV2d & KY810325 \\
HID5734 & Icheon & 2016 & NA & 705 & PCV2d & MG715490 \\
HID5735 & Jeju & 2016 & NA & 702 & PCV2b & MG715491 \\
HID5736 & Nonsan & 2017 & NA & 705 & PCV2d & MG715492 \\
HID5737 & Nonsan & 2017 & NA & 705 & PCV2d & MG715493 \\
HID5738 & Nonsan & 2017 & NA & 705 & PCV2d & MG715494 \\
HID5739 & Nonsan & 2017 & NA & 705 & PCV2d & MG715495 \\
HID5740 & Nonsan & 2017 & NA & 705 & PCV2d & MG715496 \\
HID5741 & Nonsan & 2017 & NA & 705 & PCV2d & MG715497 \\
HID5742 & Nonsan & 2017 & NA & 705 & PCV2d & MG715499 \\
\hline
\end{tabular}

ORF, open reading frame; nt, nucleotide; NA, not available.

*Corresponding author

Tel: +82-33-250-8671, Fax: +82-33-259-5625

E-mail: twhahn@kangwon.ac.kr 
PCV2 has a genome of 1766-1768 nucleotides in length that contains at least four major open reading frames (ORFs). The largest ORF1 encodes proteins (Rep and Rep') essential for viral replication in host cells. ORF2 encodes a structural capsid (Cap) protein, which is the immunodominant region inducing neutralizing antibody in hosts. ORF3 and ORF4 encode nonstructural proteins associated with apoptotic and anti-apoptotic activity in PCV2-infected cells, respectively.

PCV2 can be classified into five genotypes (PCV2a, b, c, $\mathrm{d}$, and e) based on the ORF2 sequence. The first identified PCV2a was the most prevalent genotype in pigs from 1996 to the early 2000s [1]. Since 2005, PCV2b has been reported to be the dominant genotype associated with PCVAD in pigproducing countries [12]. The newly emerging PCV2d (formerly mutant PCV2b) has been recognized in the USA, Korea, China, Germany, and Brazil [13]. In Korea, PCV2 was first identified in pigs showing PMWS [2]. PCV2a circulated in Korean pig farms until a genotype shift occurred from PCV2a to PCV2b in 2002 [7]. PCV2d is currently the predominant genotype although major PCV2 genotypes (PCV2a and PCV2b) are still being detected in the Korean pig population [8].

PCV2 Cap protein is the major immunogenic protein that induces protective immunity against PCV2 infection and plays an important role in binding to a receptor, heparan sulfate [10]. The ORF2 gene of PCV2a has a high level of nucleotide similarity with that of PCV2b, but is significantly different from that of PCV2d, which spread rapidly worldwide [11]. To allow molecular diagnosis and vaccine development, further studies are required to understand the genetic variation of the ORF2 gene associated with production of neutralizing antibodies against PCV2 genotypes. In this study, we sequenced on either full-length or ORF2 gene of PCV2 isolates in Korea from 2016 to 2017, and investigated the heterogeneity in the ORF2 gene of the PCV2 isolates.

A total of 244 blood and saliva samples were randomly selected from 23 pig farms in Korea from 2016 to 2017. The geographic locations of the PCV2 isolates used in this study are summarized in Table 1. All samples were centrifuged for $10 \mathrm{~min}$ and the supernatants were collected and stored at $-80^{\circ} \mathrm{C}$ until further use. Viral DNA was extracted from $300 \mu \mathrm{L}$ of samples using the Viral Gene-spin DNA/RNA Extraction Kit (iNtRON Biotechnology, Korea) according to the manufacturer's instructions. PCR was performed in total volume $20 \mu \mathrm{L}$ reaction mixture containing $1 \mu \mathrm{L}$ of viral DNA, $1 \mu \mathrm{L}$ of $10 \mathrm{pM}$ of each of two primers (ORF1: PCV2F1, 5'-ACCAGCGCACTTCGGCAG-3'; PCV2-R976, 5'-GG AAATTCAGGGCATGGGGG-3' and ORF2: PCV2-F867, 5'GCTCTCTATCGGAGGATTAC-3'; PCV2-R1768, 5'-AATAC TTACAGCGCACTTCTTTCG-3'), TOPsimple DyeMIX-Tenuto (Enzynomics, Korea), and $\mathrm{dH}_{2} \mathrm{O}$. The $\mathrm{PCR}$ products were ligated into RBC TA Cloning Vector (RBC Bioscience, Taiwan) and transformed into competent $E$. coli DH5 $\alpha$ cells. The correct clones having the cloned plasmid DNA were selected and the nucleotide sequences were determined with

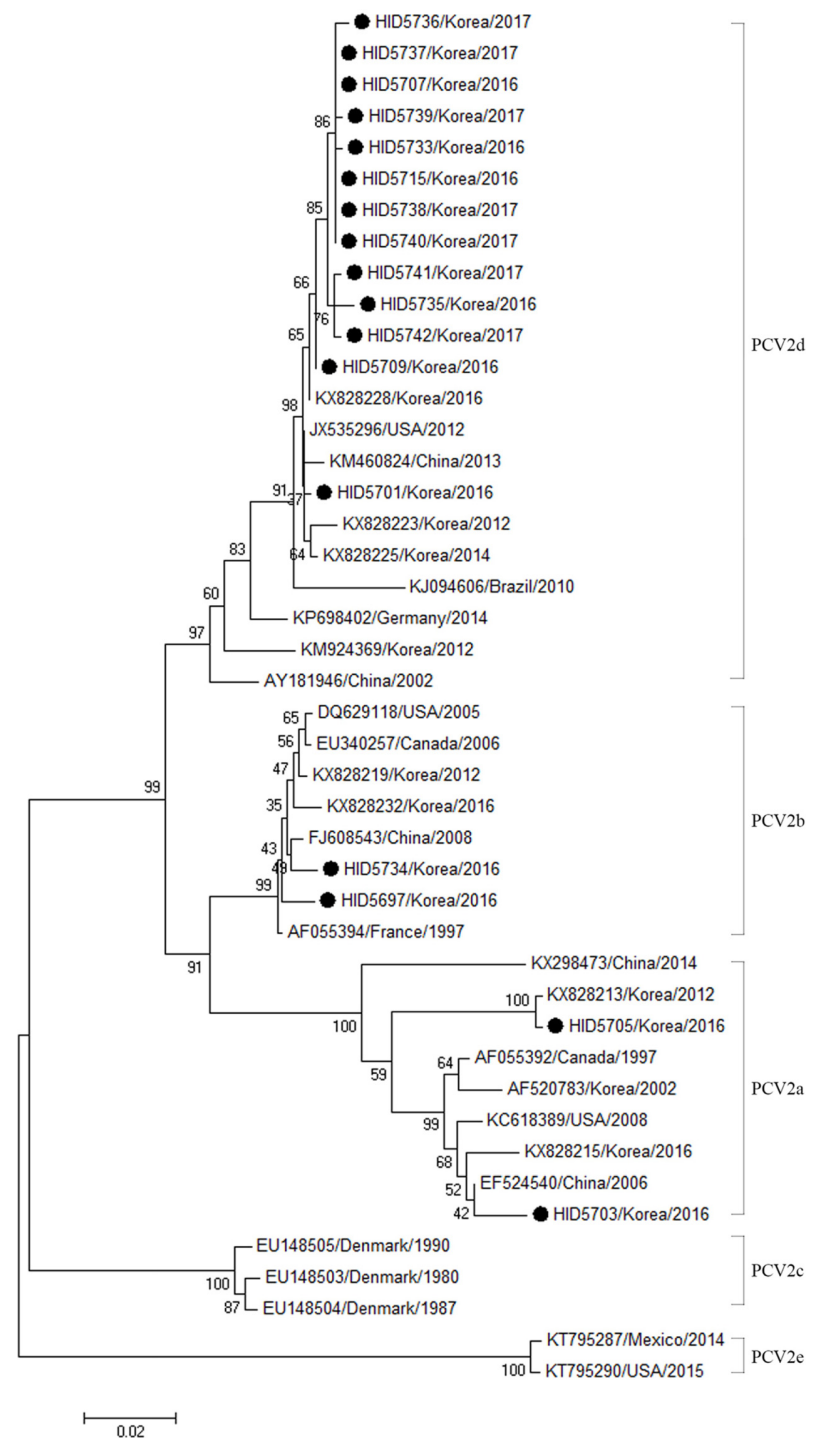

Fig. 1. Phylogenetic analysis of the Korean PCV2 isolates based on the nucleotide sequences of the ORF2 gene. Multiplesequencing alignment was performed using ClustalW [9], and the phylogenetic tree was obtained using the neighbor-joining method in the MEGA 6.0 software [15]. The numbers at each branch represent bootstrap values greater than $50 \%$ of 1,000 replicates. The scale bar indicates 0.02 nucleotide substitutions per site.

M13 forward and reverse primers (Cosmo Genetech, Korea). For phylogenetic analysis, the ORF2 genes of Korean PCV2 isolates were compared with 27 reference PCV2 sequences deposited in GenBank (National Center for Biotechnology Information, USA). A multiple sequence alignment was generated using the ClustalW program [9]. Phylogenetic tree was constructed by the neighbor-joining method and bootstrap analysis with 1,000 replicates using MEGA 6.0 software [15].

The full-length genomes $(n=7)$ or ORF2 genes $(n=10)$ of PCV2-positive samples from 8 pig farms were successfully sequenced. The ORF2 nucleotide sequences from the PCV2 

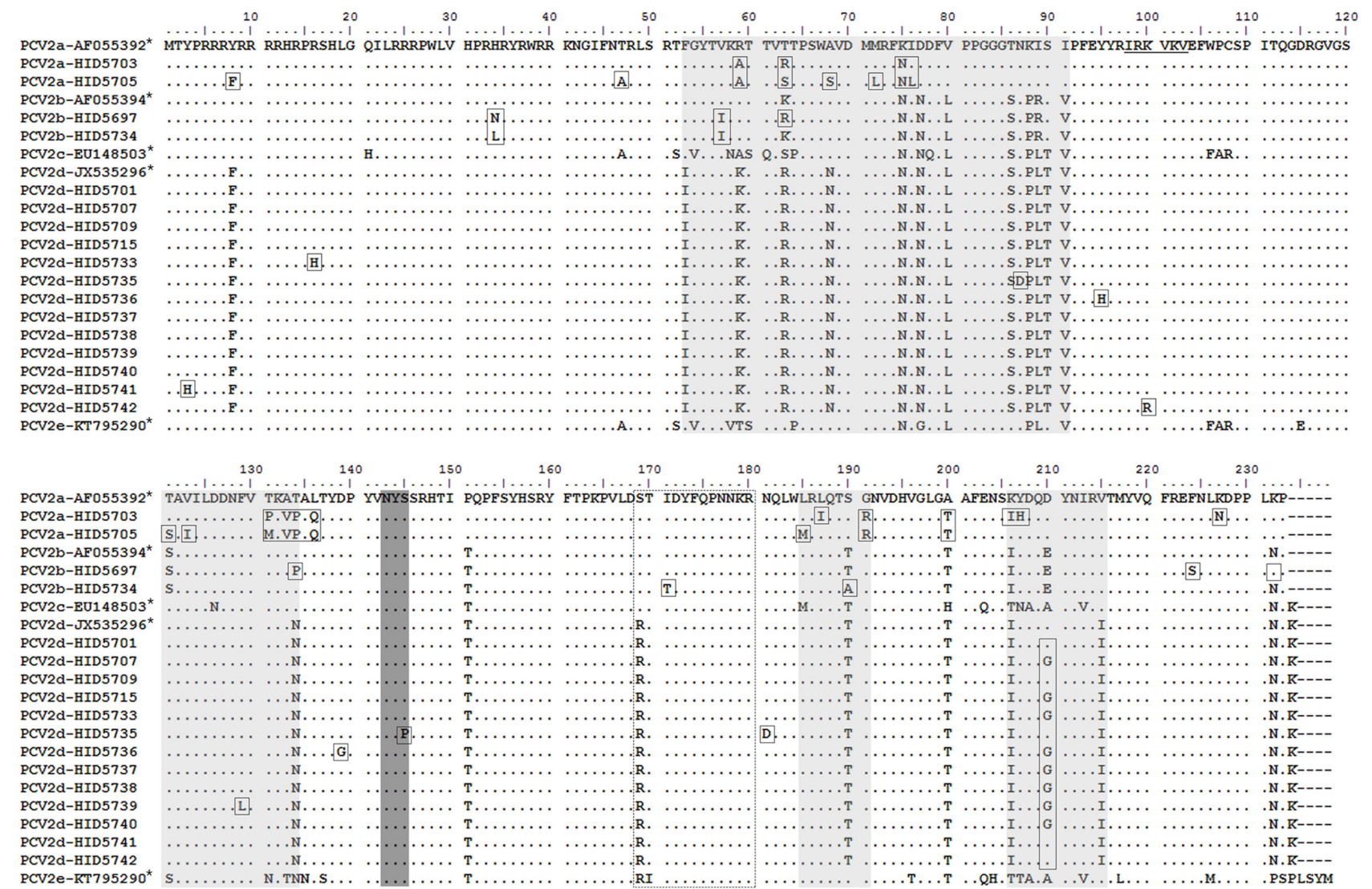

Fig. 2. Alignment of deduced amino acid sequences based on the ORF2 gene. Asterisks show the representative PCV2a, PCV2b, PCV2c, PCV2d and PCV2e genotypes. The open boxes indicate amino acid variations between Korean PCV2 isolates and representative PCV2 genotypes. Potential hypervariable domains are shown in light grey areas, and a dark grey area indicates potential sites of $N$-glycosylation [3]. The dashed line box represents the immunodominant decoy epitope [18]. The underline corresponds to the putative heparan sulfate-binding receptor domain described in Misinzo et al. [10].

isolates consisted of 702 or $705 \mathrm{bp}$. The ORF2 genes of the PCV2d isolates were three nucleotides (705 bp) longer than that of the PCV2a and PCV2b isolates. The degree of identity of ORF2 from the 17 PCV2 isolates ranged from $88.3 \%$ to $100 \%(86.3 \%$ to $100 \%)$ at the nucleotide (deduced amino acid) level. Among the 17 isolates, 2 (11.8\%), 2 (11.8\%), and 13 (76.4\%) were determined as PCV2a, PCV2b, and PCV2d, respectively, based upon the DNA sequencing of ORF2 (Fig. 1). This results indicate that PCV2d is currently spreading rapidly in the Korean swine populations, which support that the genotype shift from PCV2a and $2 b$ to PCV2d occurred on a nationwide scale reported by [8].

The similarity analysis of the 17 PCV2 isolates and representative PCV2 genotypes showed that the ORF1 gene was conserved, while the ORF2 gene was highly variable (data not shown). By multiple sequence alignment of deduced amino acid sequences, our results indicated that the ORF2 gene exhibited at least four major heterogenic regions at residues 53-91, 121-134, 185-191, and 206-215 (Fig. 2). Especially, these heterogenic regions were overlapped with the antibody recognition domains (residues 51-84, 113-132, 161208, and 228-233) described by [17]. These results may reflect an effective strategy of PCV2 to escape from host immune responses.

There is considerable interest in the neutralizing epitope of the Cap protein that is a potential target for vaccine design. Recently, selection pressures of Korean PCV2 were identified in Cap protein (position 30, 59, 80, 131, 133, 190, and 232) at a significant level [8]. The alanine at amino acid position 59 of the PCV2a Cap protein is critical for neutralizing activity [4]. However, our results showed that PCV2b and PCV2d had single amino acid changes at position 59 to arginine and lysine, respectively. Also, it has also been reported that conformational epitopes (Cap 231-233) could be detected by neutralizing monoclonal antibodies to PCV2 [14]. In this study, PCV2a had amino acid residues L-K-P at positions 231-233, while the sequence is $\mathrm{L}-\mathrm{N} / \mathrm{K}-\mathrm{P}$ in $\mathrm{PCV} 2 \mathrm{~b}$ strains. PCV2c and PCV2d had amino acid sequence $\mathrm{L}-\mathrm{N}-$ $\mathrm{P}-\mathrm{K}$ because of the addition of one amino acid (lysine, $\mathrm{K}$ ) at position 234. In addition, compared with the representative PCV2 genotypes, amino acid substitutions were observed in the PCV2a isolates (Y8F, T47A, R59A, T63R/S, A68S, M72L, K75N, I76L, T121S, V123I, T131P/M, A133V, T134P, L136Q, L185M, L187I, G191R, A200T, K206I, Y207H and K227N), 
PCV2b isolates (H34N/L, V57I, K63R, T134P, I171T, T190A, F224S and N232K), and PCV2d isolates (Y3H, R16H, N87D, Y95H, K100R, F129L, D139G, S145P and N181D). Interestingly, $61.5 \%(8 / 13)$ of PCV2d isolates had glycine at position 210 , which has not been previously reported. The results of this study indicate that genetic evolution of Cap protein may allow virus to escape neutralizing antibodies induced by commercial vaccines based on PCV2a in pigs.

An immunological decoy exists in hypervariable regions such as glycoprotein 120 of human immunodeficiency virus and glycoprotein 5 of porcine reproductive and respiratory syndrome virus [19]. However, the PCV2 decoy epitope, 169-STIDYFQPNNKR-180, is highly conserved among almost all PCV2 [18]. Vaccination with Cap (169-180) monomer elicited strong antibody response to the decoy epitope, however, only a low level of virus-neutralizing activity was detected, resulting in viremia in response to PCV2 challenge similar to that in non-immunized pigs [19]. Also, the commercial PCV2 vaccine containing lower decoy epitope induced a significantly higher level of neutralizing antibody after immunization in guinea pigs [5]. In this study, we found a single amino acid substitution in the decoy epitope of a PCV2b isolate, isoleucine (I) to threonine (T) at position 171. This result may enhance antibody production to divert the host humoral immune response away from neutralizing activity against PCV2 infection.

In summary, our study revealed that major PCV2 genotypes (PCV2a, PCV2b, and PCV2d) co-circulate and that $\mathrm{PCV} 2 \mathrm{~d}$ is currently the predominant genotype in the Korean pig population. Furthermore, all PCV2 isolates are continually changing their Cap protein including multiple antibody recognition domains. Therefore, these data provide useful information for improving the epitope-based vaccine and molecular diagnosis against current circulating PCV2 genotype.

\section{Acknowledgments}

This study was supported by Institute of Veterinary Science, Kangwon National University, Korea.

\section{References}

1. Allan G, Krakowka S, Ellis J, Charreyre C. Discovery and evolving history of two genetically related but phenotypically different viruses, porcine circoviruses 1 and 2. Virus Res 2012, 164, 4-9.

2. Choi C, Chae C. In-situ hybridization for the detection of porcine circovirus in pigs with postweaning multisystemic wasting syndrome. J Comp Pathol 1999, 121, 265-270.

3. Cooper CA, Gasteiger E, Packer NH. GlycoMod-a software tool for determining glycosylation compositions from mass spectrometric data. Proteomics 2001, 1, 340-349.

4. Huang LP, Lu YH, Wei YW, Guo LJ, Liu CM. Identification of one critical amino acid that determines a conformational neutralizing epitope in the capsid protein of porcine circovirus type 2. BMC Microbiol 2011, 11, 188.
5. Jin J, Park C, Cho SH, Chung J. The level of decoy epitope in PCV2 vaccine affects the neutralizing activity of sera in the immunized animals. Biochem Biophys Res Commun 2018, 496, 846-851.

6. Kedkovid R, Woonwong Y, Arunorat J, Sirisereewan C, Sangpratum N, Kesdangsakonwut S, Tummaruk P, Teankum K, Assavacheep P, Jittimanee S, Thanawongnuwech R. Porcine circovirus type 3 (PCV3) shedding in sow colostrum. Vet Microbiol 2018, 220, 12-17.

7. Kim D, Ha Y, Oh Y, Chae C. Prevalence of porcine circovirus types $2 \mathrm{a}$ and $\mathrm{b}$ in pigs with and without postweaning multi-systemic wasting syndrome. Vet J 2011, 188, 115-117.

8. Kwon T, Lee DU, Yoo SJ, Je SH, Shin JY, Lyoo YS. Genotypic diversity of porcine circovirus type 2 (PCV2) and genotype shift to PCV2d in Korean pig population. Virus Res 2017, 228, 24-29.

9. Larkin MA, Blackshields G, Brown NP, Chenna R, McGettigan PA, McWilliam H, Valentin F, Wallace IM, Wilm A, Lopez R, Thompson JD, Gibson TJ, Higgins DG. Clustal $\mathrm{W}$ and Clustal $\mathrm{X}$ version 2.0. Bioinformatics 2007, 23, 2947-2948.

10. Misinzo G, Delputte PL, Meerts P, Lefebvre DJ, Nauwynck HJ. Porcine circovirus 2 uses heparan sulfate and chondroitin sulfate B glycosaminoglycans as receptors for its attachment to host cells. J Virol 2006, 80, 3487-3494.

11. Olvera A, Cortey M, Segalés J. Molecular evolution of porcine circovirus type 2 genomes: phylogeny and clonality. Virology 2007, 357, 175-185.

12. Opriessnig T, Xiao CT, Gerber PF, Halbur PG. Emergence of a novel mutant PCV2b variant associated with clinical PCVAD in two vaccinated pig farms in the U.S. concurrently infected with PPV2. Vet Microbiol 2013, 163, 177-183.

13. Ramos N, Mirazo S, Castro G, Arbiza J. First identification of porcine circovirus type $2 \mathrm{~b}$ mutant in pigs from Uruguay. Infect Genet Evol 2015, 33, 320-323.

14. Shang SB, Jin YL, Jiang X, Zhou JY, Zhang X, Xing G, He JL, Yan Y. Fine mapping of antigenic epitopes on capsid proteins of porcine circovirus, and antigenic phenotype of porcine circovirus type 2. Mol Immunol 2009, 46, 327334.

15. Tamura K, Stecher G, Peterson D, Filipski A, Kumar S. MEGA6: Molecular Evolutionary Genetics Analysis version 6.0. Mol Biol Evol 2013, 30, 2725-2729.

16. Tischer I, Gelderblom H, Vettermann W, Koch MA. A very small porcine virus with circular single-stranded DNA. Nature 1982, 295, 64-66.

17. Trible BR, Kerrigan M, Crossland N, Potter M, Faaberg K, Hesse R, Rowland RRR. Antibody recognition of porcine circovirus type 2 capsid protein epitopes after vaccination, infection, and disease. Clin Vaccine Immunol 2011, 18, 749-757.

18. Trible BR, Rowland RRR. Genetic variation of porcine circovirus type 2 (PCV2) and its relevance to vaccination, pathogenesis and diagnosis. Virus Res 2012, 164, 68-77.

19. Trible BR, Suddith AW, Kerrigan MA, Cino-Ozuna AG, Hesse RA, Rowland RRR. Recognition of the different structural forms of the capsid protein determines the outcome following infection with porcine circovirus type 2. J Virol 2012, 86, 13508-13514. 